\title{
The ultrasound-guided Erector Spinae Plane Block (ESPB) allows opioid free anesthesia (OFA) in the total mastectomy with axillary dissection: a Pilot study about 14 cases
} \author{
S. Elayoubi, A. Ghannam, B. El Ahmadi, Z. Belkhadir.
National institute of oncology, Rabat, Morocco

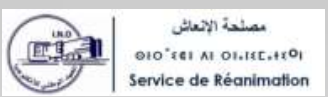

Introduction :

Total mastectomy with axillary dissection is common in the surgical management of breast cancer. Usually carried out under balanced general anesthesia using opiates, it is a source of postoperative nausea, moderate to severe postoperative acute pain with a risk of chronic pain in 25 to $60 \%$ of cases. Opioid-free anesthesia (OFA) aims to reduce the undesirable effects of opioids while ensuring optimal anesthesia and analgesia perioperatively. We report the experience of the anesthesia and resuscitation department of the National Institute of Oncology in the implementation of an opioid-free anesthesia protocol in breast cancer surgery based on ESPB.

\section{Material and method :}

We recruited for 1 month, in pre-anesthetic consultation, patients scheduled for total mastectomy with axillary dissection, class ASA I and II, presenting no contraindications to the anesthetic technique and consenting after information. The opioid-free anesthesia protocol included standard bispectral index monitoring, propofol-based intravenous induction $(2.5 \mathrm{mg} / \mathrm{kg}$ ) without curarization and an ultrasound-guided erector spinae plane block with $20 \mathrm{ml}$ of $0.25 \%$ bupivacaine at T4 level. A supra-glottic device was put in place for controlled assisted ventilation. Prior to incision, patients received a bolus of $0.5 \mathrm{mg} /$ kg Ketamine IV, 100 mg Ketoprofen IV, 8 mg dexamethasone IV and $1 \mathrm{~g}$ paracetamol IV before waking. Maintenance of anesthesia was performed by sevoflurane at $1 \mathrm{MAC}$. In case of haemodynamic repercussions related to nociception, a bolus of fentanyl at $3 \mu \mathrm{g} / \mathrm{kg}$ was administered. The parameters collected were epidemiological, oncological, anesthetic and analgesic in the perioperative period.

Table 1: Postoperative pain assessment data in our patients

\begin{tabular}{|c|c|c|c|c|c|c|c|}
\hline $\mathrm{N}$ & VAS at $\mathrm{MR}$ & $\begin{array}{l}\text { vas at } \\
\text { Ht2 }\end{array}$ & $\begin{array}{l}\text { Yas at } \\
\text { H24. }\end{array}$ & ON24 at & PONV & $\begin{array}{l}\text { Morphing post } \\
\text { op (ma) }\end{array}$ & $\begin{array}{l}\text { Sathataction at } \\
\text { H24 }\end{array}$ \\
\hline 1 & 3 & 2 & 2 & 0 & 0 & 0 & 8 \\
\hline 2 & 3 & 2 & 2 & 0 & 0 & 0 & 8 \\
\hline 3 & 3 & 2 & 2 & 0 & 0 & 0 & 8 \\
\hline 4 & 2 & 2 & 3 & 0 & 0 & 0 & 6 \\
\hline 5 & 3 & 2 & 3 & 1 & 0 & 0 & 7 \\
\hline 6 & 1 & 2 & 3 & 0 & 0 & 0 & 5 \\
\hline 7 & 5 & 3 & 2 & 1 & 0 & 3 & B \\
\hline 8 & 1 & 2 & 2 & 0 & 0 & 0 & 9 \\
\hline 9 & 3 & 2 & 1 & 1 & 0 & 0 & 9 \\
\hline 10 & 1 & 3 & 2 & 0 & 0 & 0 & 9 \\
\hline 11 & 1 & 1 & I & 2 & 0 & 0 & 6 \\
\hline 12 & 1 & 2 & 2 & 3 & 0 & 0 & 5 \\
\hline 13 & 2 & 1 & 2 & 0 & 0 & 0 & 8 \\
\hline 14 & t & 2 & 2 & 1 & 0 & 0 & 7 \\
\hline
\end{tabular}

\section{Results :}

We included 14 of the 18 recruited patients (4 refusals for regional anesthesia). The median age was 48 years, the median BMI was $26 \mathrm{~kg} \mathrm{/} \mathrm{m}^{2}$. 13 patients / 14 were ASA 1. All patients were anesthetized without modification of the protocol described. Hemodynamic variations were all less than $20 \%$ of baseline. No patient had any intraoperative analgesia supplemented by an opiate. Upon waking, patients had a median visual analogical scale (VAS) $<3 / 10$. During the first 24 hours, out of the 98 VAS surveys performed, in 94 cases the response was less than $3 / 10$. No cases of PONV were noted. Only one patient received a bolus of $3 \mathrm{mg}$ morphine IV as part of the catch-up analgesia. At $24 \mathrm{~h}$, all patients had no resting pain. 5 out of 14 patients had low mobilization pain without hyperalgesia. The DN4 score was $<4 / 10$ in all patients. Overall satisfaction with analgesia had a median of $8.5 / 10$. (table 1 )

\section{Discussion :}

Opioid-free anesthesia, which is of major interest in breast surgery, is used to avoid short-term and long-term adverse effects of opioids, such as: nausea, postoperative hyperalgesia, chronic pain and tumor recurrence. The ESPB allows to practice anesthesia and analgesia in a safe way and with high quality, avoiding the para vertebral block complications and the anterior Serratus Block variable efficiency.

Several studies have reported the efficacy of ESPB in the management of postoperative multimodal analgesia.

The Bing Fang team reported that there was no significant difference between ESPB and para-vertebral block in thoracic surgery regarding postoperative pain. A review of the literature carried out by the team of J. Caruzo interesting 242 clinical case of ESPB, between 2016 and 2018, reports the effectiveness and safety of this block in different surgeries (thoracic, abdominal and orthopedic)

Our study suggests the efficacy of ESPB in major breast surgery for opioid-free anesthesia (OFA). The study is still ongoing to confirm statistically these findings. Prospective and comparative cohort studies are needed to support these clinical findings.

References:

Bin Fang. Et al. US-guided preop single-dose ESPB provides comparable analgesia to thoracic PVB Ann Transl Med. 2019

J. Caruzo et al. The erector spinae plane (ESP) block: A pooled review of 242 cases. Med. Cureus. 2019 